\title{
Flow Field Analysis in The Domain of Work of Turbine Applied to Submunition Fuze
}

\author{
XU Sheng-gang \\ Key Laboratory of National Defense Science and Technology \\ for Mechatronics Engineering and Control \\ Beijing Institute of Technology, BIT \\ Beijing, China \\ xushgang@gmail.com \\ FAN Ning-jun \\ Key Laboratory of National Defense Science and Technology \\ for Mechatronics Engineering and Control \\ Beijing Institute of Technology, BIT \\ Beijing, China
}

\begin{abstract}
According to the requirements of United Nations on cluster munition submunition, this paper provides a kind of MEMS(micro-electromechanical Systems) suitable for the fuze, which uses turbine as the power of submunition fuze, and analyses flow field of turbine in the domain of work with simulation. The velocity of flow field of turbine in the domain of work is founded smaller with the original outline dimension of submunition through analysis, which can't meet the requirement of power generation. An improvement of the outline dimension of submunition is carried on in this paper for the purpose of cutting down the step size near the work field of turbine. The fact discovered through the simulation is that the reduction in measurement of step can improve the speed of flow field of turbine in the domain of work. Moreover, the project of reduction in width of the top size of step is superior to the other projects in the improvement.
\end{abstract}

Keywords-submunition; MEMS; turbine; flow field in the domain of work

\section{INTRODUCTION}

The presence of cluster munition dud can bring a huge casualty to common people. America used a lot of submunitions in the Gulf War, Afghanistan and Iraq War, and left numbers of double use Submunitions, cause a large number of casualties. The United stations "Convention on Certain Conventional Weapons" in the draft protocol cluster ammunition regulate that the rate of dud is not to exceed $1 \%$ in cluster ammunition produced after January 1,1990,which should have the ability of self-destruction, self-neutralization and self-disability, and external transfer must meet the above conditions. The previous research can prove that traditional mechanical fuze can't satisfy that special requirement of rate, therefore, a kind of mechanical-electrical fuze should be developed, which will use the ballistic environment of submunition to generate power, and reduce the rate of dud by electric self-destruction. Power supply is the key in this project.

\author{
WU Yan-xuan \\ Key Laboratory of National Defense Science and Technology \\ for Mechatronics Engineering and Control \\ Beijing Institute of Technology, BIT \\ Beijing, China
}

\author{
CHENG Dong-qing \\ Key Laboratory of National Defense Science and Technology \\ for Mechatronics Engineering and Control \\ Beijing Institute of Technology, BIT \\ Beijing, China
}

More and more new kinds of domestic and overseas conventional ammunition fuze adopt fuze physical power, and present the advantages of the technology in different application fields. The fuze physical power includes a wide range of pneumatic turbine generator, jet generator, ring generator and so on. On the one hand turbine can supply the needed electric energy in the system design, on the other hand it can provide necessary environmental motivation for fuze remove insurance institutions. This is a kind of electromechanical device with mature technology and wide application, which has become the most classical fuze physical power supply. Turbine should be used as the physical power supply of submunition fuze with regard to the characteristics of submuniton structure, the volume of power supply is small, and it generates electricity by circumfluence field environment of Submunition to supply power for submunition fuze.

\section{CHARACTERISTIC ANALYSIS OF CIRCUMFLUENCE FIELD OF SUBMUNITION}

The diagram of turbine suitable for submunition fuze is described in Fig. 1.

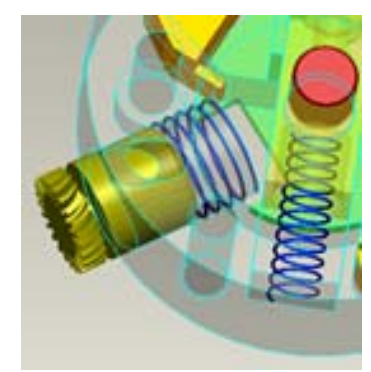

Fig. 1 Diagram of turbine suitable for submunition fuze

In the project, the turbine hides inside fuze without work, pops out in the process of submunition falling down and

Here, I am deeply grateful to my tutor, Professor FAN Ning-jun, who has checked through my thesis with patience and given me instructive suggestions. Then thanks to my teacher-WU Yan-xuan, and Dr CHENG Dong-qing, who have discussed with me about my thesis. 
generates electricity by circumfluence field environment of submunition.

The circumfluence field environment of submunition should be studied since submunition physical power supply use it to generate electricity.

Firstly, three-dimensional model is created on submunition. Secondly, FLUENT are used to help to do flow field analysis of submunition structure(FLUENT is the special CFD software for simulation and analysis of fluid flow in the complex geometric area).Discrete equation should use pressure based couple algorithm and open second order wind model to improve precision in the numerical simulation; pressure far field condition should be applied to boundary, Mach number(falling speed of submunition) and the rotate speed of Submunition are set in the import, export is set to natural outflow, the wall use no slip condition and Spalart-Allmarass turbulent model should be enabled in the analysis.

The mean falling speed of Submunition is $30 \sim 50 \mathrm{~m} / \mathrm{s}$, here $50 \mathrm{~m} / \mathrm{s}$ is selected, rotate speed is 2000rpm,angle of attack is 10 - The obtained velocity distribution of circumfluence field environment of Submunition is depicted in Fig. 2.

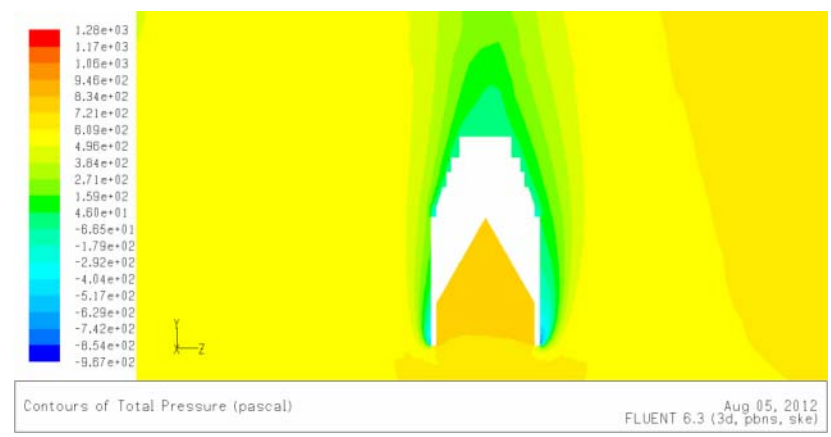

Fig. 2 Velocity distribution of circumfluence field environment of Submunition

As described in Fig. 2, the velocity of circumfluence field environment of Submunition is smaller than velocity of the area slightly far from Submunition wall, and moreover the velocity increases as the distance from the wall increases.

\section{FLOW FILED VELOCITY DISTRIBUTION ANALYSIS IN THE DOMAIN OF WORK OF TURBINE}

Flow field environment of turbine work area should be analyzed in order to obtain the velocity distribution, flow field in the six lines of submunition circumfluence are selected according to fuze pop-up physical power supply (see Fig. 3).

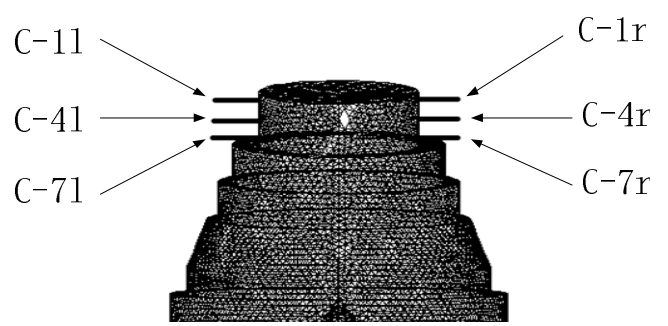

Fig. 3 Locations of selected six lines of submunition circumfluence
As shown in Fig. 3, C-1l,C-4l, C-7l, C-1r, C-4r, C-7r are six lines. C-4l, C-1l, C-7l are the locations of center axis, impeller upper edge and lower edge of turbine installed on the left side, respectively. C-4r, C-1r, C-7r are the locations of center axis, impeller upper edge and lower edge of turbine installed on the right side, respectively.

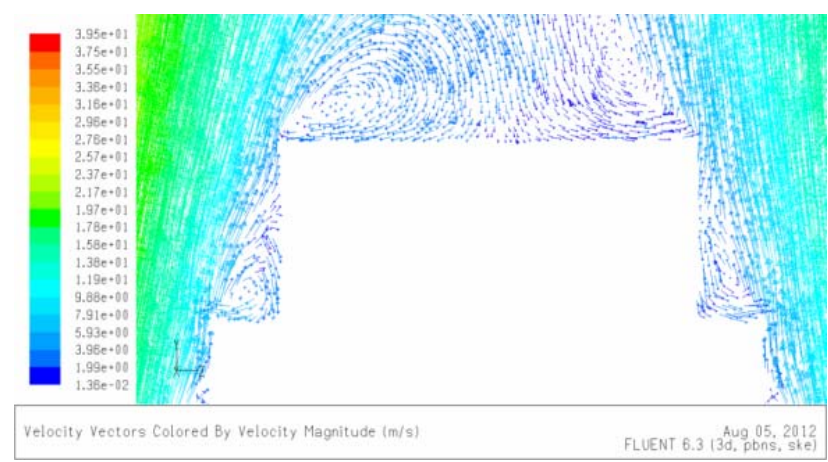

Fig. 4 The illustration of velocity vector of turbine in the domain of work

The fact founded in the Fig. 4 is that within the range of closer distance from the wall, the velocity of flow field is small and in the step whirlpool appears, and the velocity becomes negative.

Velocity distributions are drawn in Fig. 5 and Fig. 6 with analysis at a distance of $0 \sim 5 \mathrm{~mm}$ from wall in the six lines.

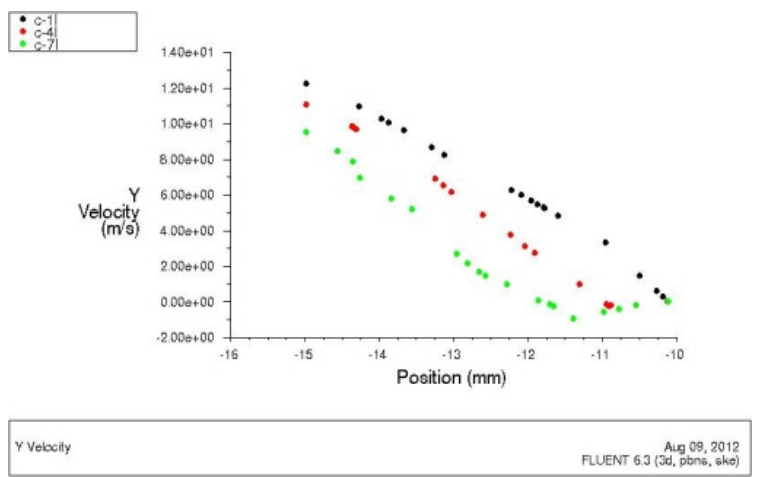

Fig. 5 Velocity distribution in the three lines on the left side

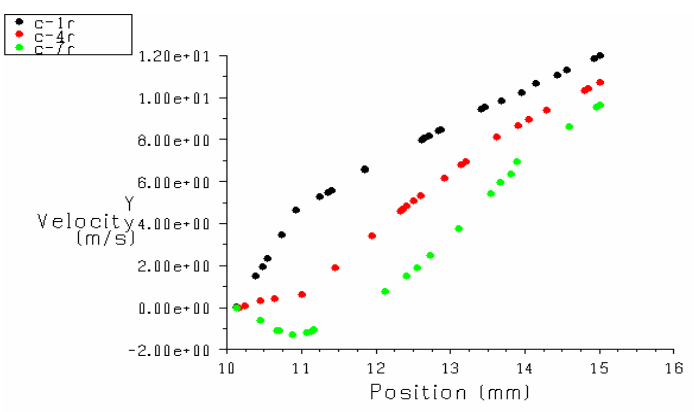

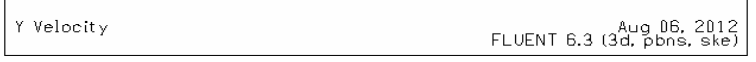

Fig. 6 Velocity distribution in the three lines on the right side

The velocity of turbine in the domain of work distributes basic symmetrically in the symmetric positions of submunition's left and right as described in Fig. 5 and Fig. 6. 
Since the angle of attack of $10^{\circ}$ exists, velocity of left is a little larger than the right in the symmetric position; the direction of velocity will reverse in the position line of lower of turbine at a distance of $1 \mathrm{~mm}$ from the wall. The closer to the upper position of submunition, the larger velocity of circumfluence field will become; velocity of flow field has a relationship to the distance from the wall within a range of $0 \sim 5 \mathrm{~mm}$, it increases basically along with the increasement of the distance from the wall.

Velocity of flow field is small within the work area of turbine. At a distance of $5 \mathrm{~mm}$ from wall, the velocity in the center axis of turbine is $11 \mathrm{~m} / \mathrm{s}$, it is small. Such velocity can't meet the power requirement of the whole power system through calculation. Therefore, the velocity should be raised and the distribution of the flow field velocity should be improved to meet the power requirement of submunition.

\section{MEASURES To IMPROVE THE FlOW FIELD VELOCITY IN THE DOMAIN OF WORK OF TURBINE}

This paper increases the velocity by the shape improvement of submunition. The step near whirlpool of velocity vector is one of key factors to influence the distribution of velocity of circumfluence field. This paper improves shape of step and analyses which measure to increase the velocity is most effective.

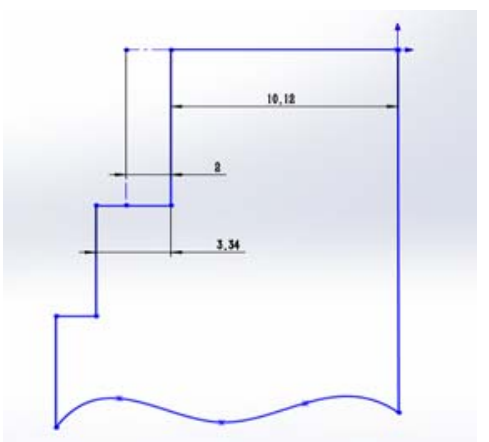

Fig. 7 The step’s size in original outline of submunition

The step's size is $3.34 \mathrm{~mm}$ in original outline of submunition(see Fig. 7).There are some projects of improvement in the size.

(1) The size reduces to $2.34 \mathrm{~mm}$, namely reduce $1 \mathrm{~mm}$ (see Fig. 8).

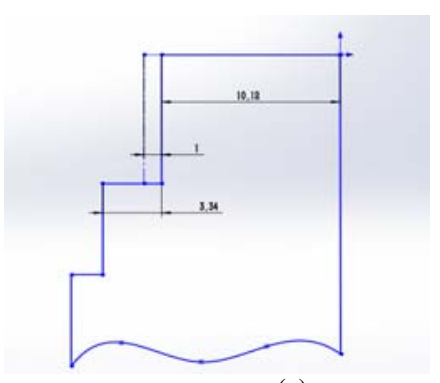

(a)

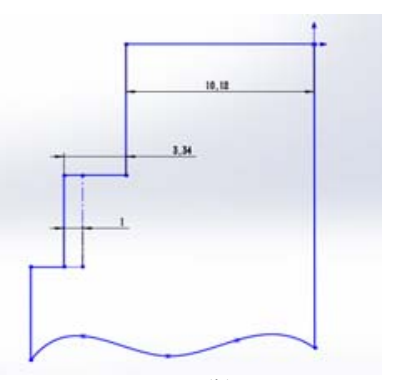

(b)
Fig. 8 The size reduces to $2.34 \mathrm{~mm}$

(2) The size reduces to $1.34 \mathrm{~mm}$, namely reduce $2 \mathrm{~mm}$ (see Fig. 9).

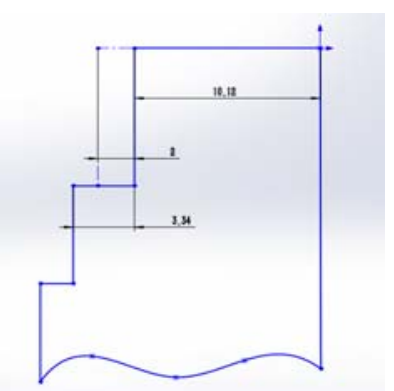

(a)

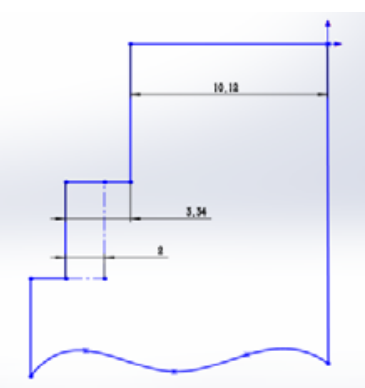

(b)

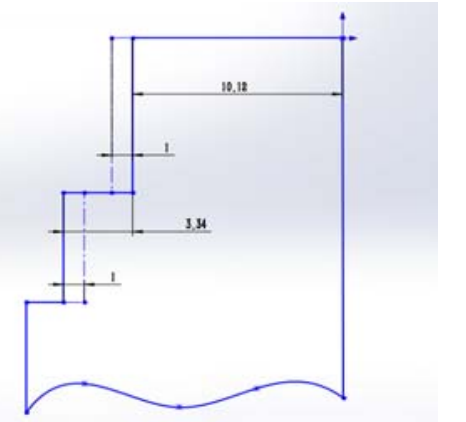

(c)

Fig. 9 The size reduces to $1.34 \mathrm{~mm}$

(3) Round off the step, the radius is $2 \mathrm{~mm}$ (see Fig. 10).

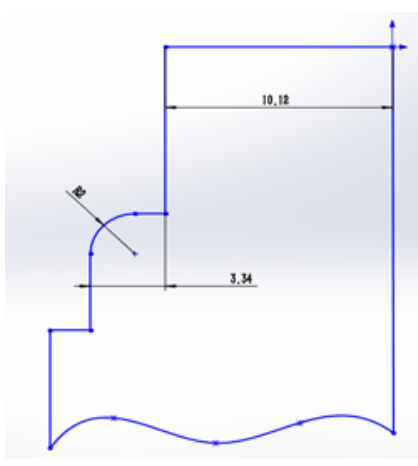

Fig. 10 Round off the step

Considering only the left side in the projects shown in Fig. $8 \sim$ Fig. 10, the distribution curve of velocity are drawn in Fig. 11.
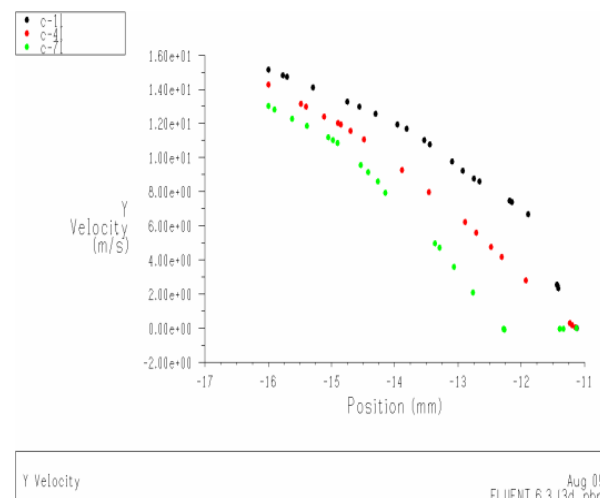

(a) 


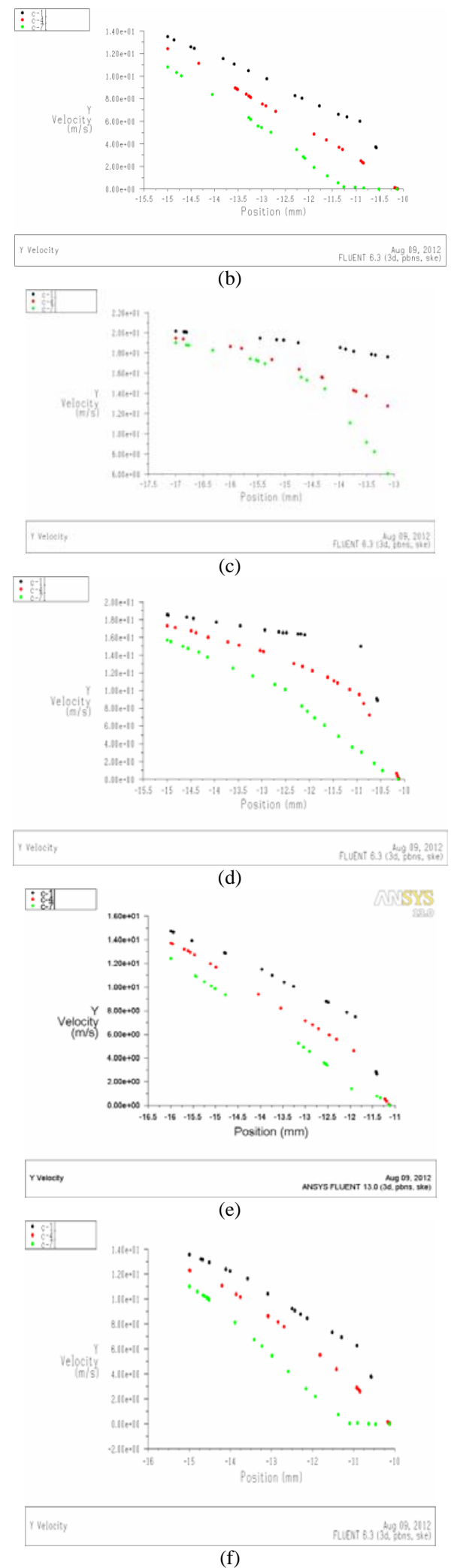

Fig. 11 Distribution curve of velocity after improvement
Some conclusions can be obtained from Fig. 11 as follows:

1) The velocity of flow field after improvement is greater than the velocity in the original project;

2) With regard to the project of reducing size to $2.34 \mathrm{~mm}$ shown in Fig. 8,the velocity of the improvement method depicted in (a) is a little larger than the velocity of the improvement method depicted in (b) in the corresponding position. The method depicted in (a) is more superior;

3) About the project of reducing size to $1.34 \mathrm{~mm}$ shown in Fig. 9, the velocity of the improvement method depicted in (a) is a little larger than the velocity of the improvement method depicted in (b) and (c) in the corresponding position. The method depicted in (a) is more superior;

4) The velocity of the improvement method depicted in Fig. 9(a) is a little larger than the velocity of the improvement method depicted in Fig. 8 and Fig. 10.The method depicted in Fig. 9(a) is more superior.

Therefore, in order to improve the flow field velocity in the domain of work of turbine, the method to improve the shape of the submunition is effective. The method depicted in Fig. 9(a) is more superior than the other methods. The reduction in width of the top size of step can improve the flow field velocity in the domain of work of turbine effectively.

\section{CONCLUSION}

This paper provides a kind of MEMS suitable for submunition fuze and analyses the flow field in the domain of work of turbine with simulation. The simulation result demonstrates that the flow field velocity in the domain of work of turbine is small under the current outline dimension of submunition, which can't meet the power requirement. This paper improves the outline dimension to increase the velocity, especially in the step near the turbine work area. The simulation result proves that the reduction in width of the top size of step can improve the flow field velocity in the domain of work of turbine effectively. This method is an effective measure to improve the flow filed in the domain of work of turbine, which has a value of further research.

\section{REFERENCES}

[1] ZHAO Yu-qing, Wang Xiao-bo, Zhang Hai-juan. Development trend of cluster ammunition convention fuze[J].Journal of Detection \& Control,2009,31:25-29.

[2] BI Jian-jun, GAO Min. US fuze technology for the 21th century[J]. Journal of Detection \& Control,1999,21(2):48-50.

[3] XUE Wei-qing. Application and development of fuze special five road power[J]. Journal of Detection \& Control,1994,16(1):8-13.

[4] LI Fu-song, YU Chun-ming, XU Min. Discussion on air driven revolving generator as velocity transducer[J]. Journal of Detection \& Control,2007,29(3):21-24.

[5] MA Bao-hua. Fuze structure and function principle[M]. Beijing: National Defence Industry Press,1984.

[6] ZHU Hong-jun. Practical tutorial of FLUENT fluid analysis and simulation[M]. Beijing: Posts \& Telecom Press,2010. 